\title{
Performance analysis of joint relay selection and transmission schemes in multiple-antenna two-way relay systems
}

\author{
Chen Chen ${ }^{1 *}$, Xingbin Wang ${ }^{2}$, Yehua Yang ${ }^{1}$ and Ye Jin ${ }^{1}$
}

\begin{abstract}
In this paper, we consider a two-way relay system with two multi-antenna sources and multiple single-antenna relays and study the performances for various transmission schemes with the assumption that perfect channel state information (CSI) is available at both the sources and the relays. Although a transmission scheme that combines source beamforming and relay selection (BF-RS) is known to improve a system performance, its performance has not been theoretically analyzed yet. In the paper, we focus on the performance analysis in terms of the symbol error probability (SEP) for the BF-RS scheme. The analytical upper and lower bounds of the end-to-end system SEP are derived in the closed form, and the asymptotic SEP expression is given in high SNR regime. By both analytical and simulation results, it is clearly shown that a full transmit and relay selection diversity gain can be achieved without code rate loss. The SEP curves from simulations show that our derived bounds can predict the performance accurately. Based on the derived analytical results, we propose an individual power allocation (IPA) scheme to save the total transmit power. We show that the IPA scheme can result in considerable energy saving with a comparable SEP performance.
\end{abstract}

Keywords: Two-way relay system; Physical-layer networking coding; Diversity gain; Power allocation

\section{Introduction}

In order to achieve a higher throughput and spectral efficiency, protocols consisting of the multiple access (MAC) and broadcast $(\mathrm{BC})$ phases in a two-way relay channel (TWRC) have attracted more research interests than conventional protocols using three or four phases. Since the physical-layer network coding (PNC) protocol can be easily applied to two-way relay systems, its performance has been widely studied [1].

For reliable transmissions, it is important to exploit diversity gain in multi-input multi-output (MIMO) wireless communications. Relay selection (RS) diversity can also be exploited in MIMO TWRC in conjunction with transmit diversity. For instance, To et al. [2] considered a two-way relay system using the Alamouti's code at the two sources while there is only one antenna at a relay.

\footnotetext{
*Correspondence: c.chen@pku.edu.cn

${ }^{1}$ State Key Laboratory of Advanced Optical Communication Systems and Networks, Peking University, 100871 Beijing, China

Full list of author information is available at the end of the article
}

Gong et al. [3] studied a system consisting of two singleantenna sources and a single relay of two-antenna. Relay and antenna selection schemes are also addressed in many related works in MIMO TWRC. Chen et al. [4] proposed a RS and beamforming scheme for cooperative two-way relay systems with the amplify-and-forward (AF) protocol. Jia et al. [5] investigated the outage probability and ergodic capacity of the two-way network coding opportunistic relaying systems with the AF protocol. Jayasinghe et al. [6] proposed a robust joint precoder-decoder design scheme for a multiple-input multiple-output PNC-based two-way relay system.

Zhou et al. [7] proposed an opportunistic two-way relay scheme based on joint network coding and opportunistic relaying with the decode-and-forward (DF) protocol. The analysis and design of space-time trellis codes (STTCs) for TWRC with multi-antenna sources and single-antenna relays were studied with the AF protocol [8].

Although various approaches for efficient transmission schemes with RS are proposed, it is interesting to note that joint transmission and RS schemes for MIMO TWRC are not well studied. In other words, the individual analysis

\section{Springer}

(c) 2015 Chen et al. This is an Open Access article distributed under the terms of the Creative Commons Attribution License (http://creativecommons.org/licenses/by/4.0), which permits unrestricted use, distribution, and reproduction in any medium, provided the original work is properly credited. 
of either the transmission schemes or the RS schemes has been well investigated, but a best combination of the transmission and RS schemes under a certain performance criterion is not known, especially in asymmetrical multi-antenna two-way relay systems. In addition, it is proved that an opportunistic RS scheme with low complexity can achieve better performance than a fully distributed two-way relay scheme [7] and it is shown that the DF protocol outperforms the AF protocol in terms of symbol error probability (SEP) performance [9]. With the demand of reliable transmissions, combining the best known techniques in multiple-antenna two-way relay systems would lead us to the best joint scheme; however, its performance is not known yet.

In addition, it is well known that the power allocation (PA) plays an important role in wireless communications. With a total power constraint, the PA is widely used to improve the system performance. With individual power constraints, the PA can be used to save the system power consumption. Song et al. [10] proposed a PA scheme based on the asymptotic SEP in AF two-way relay systems. Zhang et al. [11] proposed a PA scheme to minimize the maximum individual outage probability. Talwar et al. [12] presented an optimal joint RS and PA scheme to maximize the worst received signal-to-noise ratio (SNR) in AF two-way relay systems. Do et al. [9] proposed a joint relay selection and PA scheme to improve the SEP performance in a two-way relay system with the DF protocol. To the best of our knowledge, all these works are based on the total transmission power constraint. However, the relays and the sources could be battery-powered, which means that individual transmission power constraints at nodes are more suitable for two-way relay systems with battery-powered nodes. Hence, under individual transmission power constraints, it would be needed to consider an individual power allocation (IPA) scheme to improve the system power efficiency in a multiple-antenna twoway relay system.

In this paper, as mentioned earlier, the combination of the best known techniques for a multiple-antenna twoway relay system consisting of two sources of multiple antennas and multiple relays of single antenna is considered. A transmission scheme that combines beamforming and RS (BF-RS) is mainly considered. We present an asymptotic SEP expression for the proposed BF-RS scheme and make comparison with other transmission schemes. Through analysis and simulation results, it can be shown that a full transmit and RS diversity gain can be achieved by the BF-RS scheme. Thus, we can confirm that the BF-RS scheme is optimal in terms of the diversity gain.

Furthermore, we consider a general case where two sources are equipped with $M$ and $N$ antennas and $K$ relays participate in the transmission to derive closedform expressions for tight upper and lower bounds on SEP.
In order to improve the system power efficiency, an IPA scheme is also proposed to reduce the total transmission power cost. The performance of the BF-RS scheme and the IPA scheme is verified through simulations.

The main contributions of this paper are summarized as follows. (i) Despite that the BF-RS scheme is based on existing approaches, its performance has not been well investigated in a theoretical manner. We derive the upper and lower bounds of the end-to-end system SEP in an asymmetrical multi-antenna two-way relay system, where the source nodes are equipped with different numbers of antennas. The derived SEP expressions are tight and are verified with the simulated results. (ii) We conduct the asymptotic SEP performance analysis and evaluate the diversity order. We demonstrate that the BF-RS scheme can achieve a full transmit and RS diversity gain and outperforms other transmission and RS schemes. (iii) Under individual transmission power constraints, we propose an IPA scheme to improve the system power efficiency. The behaviors of the system power efficiency under different system configurations are investigated. Meanwhile, both simulated and analytical results show that the proposed IPA scheme can yield considerable energy saving with a comparable SEP performance to other PA schemes.

The rest of this paper is organized as follows. In Section 2, a system model of a multiple-antenna two-way relay system is introduced. In Section 3, we investigate the optimal beamforming and the RS criteria. In Section 4, an asymptotic SEP expression and the closed-form bounds are discussed. In Section 5, the IPA scheme for the BF-RS is given. Simulation results are shown to corroborate our theoretical results in Section 6. In Section 7, we draw the main conclusion.

The following notations are used in this paper. Lower and upper boldfaced letters are used for column vectors and matrices. $(\cdot)^{*}$ and $(\cdot)^{T}$ represent the conjugate and transpose, respectively. For a vector or matrix, $\|\cdot\|$ denotes the Frobenius norm. $\mathcal{C N}\left(\mu, \sigma^{2}\right)$ denotes the circularly symmetric complex Gaussian distribution with mean $\mu$ and variance $\sigma^{2}$. For a random variable (RV) $X$, $E[f(X)]$ represents the expectation of a function of $X$, $f(X) . f_{X}(\cdot)$, and $F_{X}(\cdot)$ refer to the probability density function (PDF), and the cumulative density function (CDF) of $X$, respectively.

\section{System model}

In this paper, we consider a multiple-antenna two-way relay system where two multi-antenna source nodes, $S_{1}$ and $S_{2}$, are equipped with $M$ and $N$ antennas, respectively, and $K$ relay nodes, $\left\{R_{1}, \ldots, R_{K}\right\}$ are equipped with single antenna. $S_{1}$ and $S_{2}$ exchange their information with the help of one selected relay, say $R_{k}$. This system model can be possessed in practice. For instance, two base stations equipped with multiple antennas might communicate via 
a selective single antenna relay, which is due to the size and complexity constraints. We assume that the channel state information (CSI) remains unchanged during a transmission time unit consisting of two phases (the first is the MAC phase and the second is the $\mathrm{BC}$ phase). The detailed system model is shown in Fig. 1.

In the MAC phase, the relays can receive signals from $S_{1}$ and $S_{2}$. Based on the perfect CSI assumption, the best relay $R_{k}$ out of $K$ relays is selected (the RS criteria will be discussed later). Then, the selected relay forwards the detected signals to both $S_{1}$ and $S_{2}$ in the BC phase. Using the PNC protocol, $S_{1}$ and $S_{2}$ can detect the desired information from each other. We assume that all the sources and relays operate in time division duplex mode, and perfect synchronization has been established between the sources and the relays before data transmission. In this section, we will consider a generic representation for various transmission schemes that can achieve a full transmit diversity such as beamforming, space time block coding (STBC), and so on.

We assume that the same signal constellation $\mathcal{C}$ is used at sources and relays throughout the paper. The size of $\mathcal{C}$ is denoted by $C$. In each transmission time unit, $S_{1}$ and $S_{2}$ will exchange their frames of $L$ symbols, which are denoted by $\mathbf{x}=\left[x_{1}, x_{2}, \ldots, x_{L}\right]$ and $\mathbf{y}=\left[y_{1}, y_{2}, \ldots, y_{L}\right]$, respectively. Let the operator $\Psi(\cdot)$ represent the transmission scheme and the transmission matrices $\mathbf{X}=\Psi(\mathbf{x})$ and $\mathbf{Y}=\Psi(\mathbf{y})$ be $\mathbf{X}=\left[\begin{array}{ccc}x_{11} & \cdots & x_{1 L} \\ \vdots & \ddots & \vdots \\ x_{M 1} & \cdots & x_{M L}\end{array}\right]$ and $\mathbf{Y}=$ $\left[\begin{array}{ccc}y_{11} & \cdots & y_{1 L} \\ \vdots & \ddots & \vdots \\ y_{N 1} & \cdots & y_{N L}\end{array}\right]$. For example, suppose that $M=2$ and
$L=2$. Then, we can show that each transmission scheme has a different form of $\Psi(\mathbf{x})$. For the Alamouti code [13], we have $\mathbf{X}=\Psi(\mathbf{x})$ as $\mathbf{X}=\left[\begin{array}{cc}x_{1} & -x_{2}{ }^{*} \\ x_{2} & x_{1}{ }^{*}\end{array}\right]$. For the diagonal code ([14], 6.2.22), we have $\mathbf{X}=\Psi(\mathbf{x})$ as $\mathbf{X}=\left[\begin{array}{cc}x_{1} & 0 \\ 0 & x_{1}\end{array}\right]$. For the beamforming scheme in $([14], 6.1 .12)$, we have $\mathbf{X}=\Psi(\mathbf{x})$ as $\mathbf{X}=\left[\begin{array}{ll}w_{1} x_{1} & w_{1} x_{2} \\ w_{2} x_{1} & w_{2} x_{2}\end{array}\right]$, where $w_{i}, i=1,2$ are the beamforming weights. With $\mathbf{X}$ and $\mathbf{Y}$, the received signal at $R_{k}(k=1,2, \ldots, K)$ in the MAC phase can be expressed as

$$
\mathbf{s}_{k}^{T}=\sqrt{\frac{P_{1}}{M}} \mathbf{h}_{1 k}^{T} \mathbf{X}+\sqrt{\frac{P_{2}}{N}} \mathbf{h}_{2 k}^{T} \mathbf{Y}+\Omega_{k}^{T}, k=1,2, \ldots, K,
$$

where $\mathbf{h}_{1 k}=\left[h_{11, k} h_{12, k} \ldots h_{1 M, k}\right]^{T}$ and $\mathbf{h}_{2 k}=$ $\left[h_{21, k} h_{22, k} \ldots h_{2 N, k}\right]^{T}$ are the channel vectors between relay $R_{k}$ and $S_{1}$ and relay $R_{k}$ and $S_{2}$, respectively. $\boldsymbol{\Omega}_{k} \sim$ $\mathcal{C N}\left(0, \sigma^{2} \mathbf{I}_{L}\right)$ is the background noise vector at $R_{k} . P_{m}$ is the transmit power of $S_{m}, m=1,2$.

Since the CSI is assumed to be perfectly known at $R_{k}$, under the maximum likelihood (ML) criterion, the estimate of $\mathbf{x}$ and $\mathbf{y}$, denoted by $\mathbf{x}^{\prime}$ and $\mathbf{y}^{\prime}$, is given by

$$
\left[\mathbf{x}^{\prime}, \mathbf{y}^{\prime}\right]=\underset{x_{j}, y_{j} \in \mathcal{C}}{\arg \max } \exp (-\Delta(\mathbf{X}, \mathbf{Y}))
$$

where $\Delta(\mathbf{X}, \mathbf{Y})=\left\|\mathbf{s}_{k}^{T}-\sqrt{\frac{P_{1}}{M}} \mathbf{h}_{1 k}^{T} \mathbf{X}-\sqrt{\frac{P_{2}}{N}} \mathbf{h}_{2 k}^{T} \mathbf{Y}\right\|^{2}$. For simplicity, we consider a modification of (2) in the following derivations, which does not change the final SEP results. The modified detection rule at $R_{k}$ is given by

$$
\left[\mathbf{x}^{\prime}, \mathbf{y}^{\prime}\right]=\underset{x_{j}, y_{j} \in \mathcal{C}}{\arg \min } \Delta(\mathbf{X}, \mathbf{Y}) \text {. }
$$

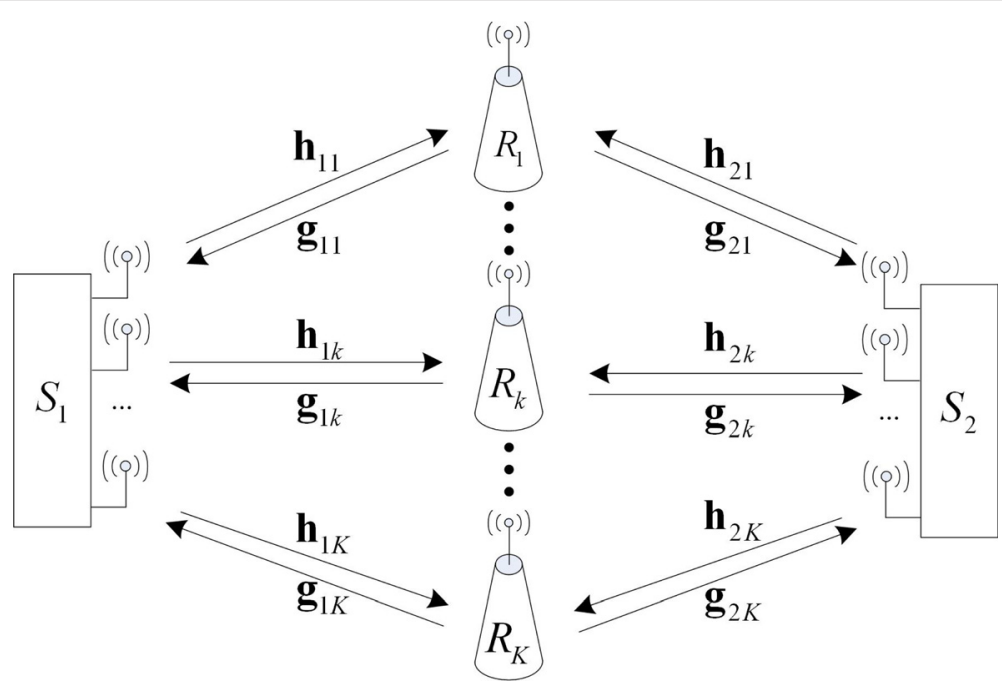

Fig. 1 The system model of a two-way relay system with multi-antenna sources and single-antenna relays 
At each relay, an XORed-like version of $\left(x_{j}, y_{j}\right), j=$ $1,2, \ldots, L$, is to be detected, which is given by

$$
z_{j}=f\left(x_{j}, y_{j}\right)=l^{-1}\left(\left(l\left(x_{j}\right)+l\left(y_{j}\right)\right),(\bmod C)\right),
$$

where $l(x)$ is considered as the mapping function [1] and $l^{-1}(x)$ represents the inverse operation of $l(x)$. The XORed-like version of $\left(\mathbf{x}^{\prime}, \mathbf{y}^{\prime}\right)$ can also be written as $\mathbf{z}^{\prime}=$ $\left[z_{1}^{\prime}, z_{2}^{\prime}, \ldots, z_{L}^{\prime}\right]=f\left(\mathbf{x}^{\prime}, \mathbf{y}^{\prime}\right)$.

In time slot $j$ of the $\mathrm{BC}$ phase, $S_{m}$ receives

$$
\mathbf{r}_{m, j}=\sqrt{P_{r}} \mathbf{g}_{m k} z_{j}^{\prime}+\mathbf{n}_{m, j}, m=1,2, j=1,2, \ldots, L,
$$

where $P_{r}$ is the transmit power at $R_{k}, \mathbf{g}_{1 k}=$ $\left[g_{11, k} g_{12, k} \ldots g_{1 M, k}\right]^{T}$ and $\mathbf{g}_{2 k}=\left[g_{21, k} g_{22, k} \ldots g_{2 N, k}\right]^{T}$ are the channel vectors between relay $R_{k}$ and $S_{1}$ and relay $R_{k}$ and $S_{3}, \mathbf{n}_{1, j} \sim \mathcal{C N}\left(0, \sigma_{1}^{2} \mathbf{I}_{M}\right)$ and $\mathbf{n}_{2, j} \sim \mathcal{C N}\left(0, \sigma_{2}^{2} \mathbf{I}_{N}\right)$ are the background noise vectors. $S_{m}$ detects $\hat{\mathbf{z}}_{m}=$ $\left[\hat{z}_{m, 1}, \hat{z}_{m, 2}, \ldots, \hat{z}_{m, L}\right], m=1,2$ as

$$
\hat{z}_{m, j}=\underset{z_{j} \in \mathcal{C}}{\arg \min }\left\|\mathbf{r}_{m, j}-\sqrt{P_{r}} \mathbf{g}_{m k} z_{j}\right\|^{2}, j=1,2, \ldots, L .
$$

Finally, each source detects the frame sent by the other source in a similar way as (4): $\hat{\mathbf{x}}=f\left(\hat{\mathbf{z}}_{2}, \mathbf{y}\right)$ and $\hat{\mathbf{y}}=f\left(\hat{\mathbf{z}}_{1}, \mathbf{x}\right)$ where $\hat{\mathbf{x}}$ and $\hat{\mathbf{y}}$ are the estimates of $\mathbf{x}$ and $\mathbf{y}$, respectively.

\section{Transmission scheme and RS criterion}

In this paper, the main objective is to investigate the best performance among various transmission schemes and RS criteria in multiple-antenna two-way relay systems. By analyzing existing transmission schemes and RS criteria, we find ${ }^{1}$ that the Alamouti code with RS schemes can only achieve a diversity gain of $d=\min (M, N)$ at a code rate of $r=1$. The diagonal code with RS schemes can achieve a full diversity gain of $d=K \min (M, N)$ at a code rate of $r=\frac{1}{\min (M, N)}$. In other words, the Alamouti code with RS and the diagonal code with RS schemes suffer performance loss in either the diversity gain or the code rate. Among the transmission schemes, beamforming schemes can be easily combined with RS and achieve the best performance. The scheme can minimize the overall system SEP and achieve a full diversity gain at a code rate of $r=1$. Thus, we focus on the performance analysis for the BF-RS scheme in this section. Prior to studying the performance of the BF-RS scheme, we first derive an expression of the system SEP.

Let $P_{\text {MAC }}^{k}$ denote the SEP in the MAC phase at $R_{k}$ and $P_{\mathrm{BC}, m}^{k}$ denote the SEP in the BC phase for given $\mathbf{h}_{1 k}$ and $\mathbf{h}_{2 k}$ at $S_{m}$. Then, we have

$$
\begin{aligned}
& P_{\mathrm{MAC}}^{k}=\operatorname{Pr}\left\{\mathbf{z}^{\prime} \neq f(\mathbf{x}, \mathbf{y}) \mid(\mathbf{x}, \mathbf{y}), \mathbf{h}_{1 \mathrm{k}}, \mathbf{h}_{2 \mathrm{k}}\right\} ; \\
& P_{\mathrm{BC}, m}^{k}=\operatorname{Pr}\left\{\hat{\mathbf{z}}_{m} \neq \mathbf{z}^{\prime} \mid \mathbf{z}^{\prime}, \mathbf{h}_{m k}\right\} .
\end{aligned}
$$

It is noteworthy that a correct detection occurs if both the phases have erroneous decisions in a two-way transmission with the BPSK modulation. Therefore, averaging the SEPs over the two phases, the instantaneous SEP at source node $S_{m}$, denoted by $P_{E \text { to } E, m}^{k}$, is given by

$$
P_{E \mathrm{to} E, m}^{k}=P_{\mathrm{MAC}}^{k}\left(1-P_{\mathrm{BC}, m}^{k}\right)+P_{\mathrm{BC}, m}^{k}\left(1-P_{\mathrm{MAC}}^{k}\right) .
$$

Thus, the instantaneous end-to-end SEP of both the source nodes $S_{1}$ and $S_{2}$, defined by $P_{E \mathrm{to} E}^{k}=\frac{1}{2} \sum_{m=1}^{2} P_{E \mathrm{to} E, m}^{k}$, is given by

$$
P_{E \mathrm{to} E}^{k}=P_{\mathrm{MAC}}^{k}+\frac{1}{2} \sum_{m=1}^{2} P_{\mathrm{BC}, m}^{k}-P_{\mathrm{MAC}}^{k} \sum_{m=1}^{2} P_{\mathrm{BC}, m}^{k} .
$$

Note that in the rest of the paper, we confine ourselves to BPSK for tractable analysis as we will focus on the performance analysis of the BF-RS scheme.

\subsection{The optimal beamforming at the two sources}

We redefine the transmission matrices at the two sources as $\mathbf{X}=\left[\begin{array}{llll}\mathbf{x}_{1} & \mathbf{x}_{2} & \ldots & \mathbf{x}_{L}\end{array}\right]$ and $\mathbf{Y}=\left[\begin{array}{llll}\mathbf{y}_{1} & \mathbf{y}_{2} & \ldots & \mathbf{y}_{L}\end{array}\right]$, where $\mathbf{x}_{j}=\mathbf{w}_{1} x_{j}$ and $\mathbf{y}_{j}=\mathbf{w}_{2} y_{j}$. Here, $\mathbf{w}_{1}=\left[\begin{array}{llll}w_{11} & w_{12} & \ldots & w_{1 M}\end{array}\right]^{T}$ and $\mathbf{w}_{2}=\left[\begin{array}{llll}w_{21} & w_{22} & \ldots & w_{2 N}\end{array}\right]^{T}$ are the beamforming vectors chosen by source $S_{1}$ and $S_{2}$. For simplicity, the time index subscript $j$ is omitted in the following derivation. We assume that the transmission power of the sources and the relays are identical, i.e., $P_{1}=P_{2}=P_{r}=P$. We also denote the SNR by $\bar{\gamma}=\frac{P}{\sigma^{2}}$, where $\sigma^{2}$ is the Gaussian noise variance. We focus on the case of $\mathbf{g}_{m k}=\mathbf{h}_{m k}, m=1,2$, by considering that the links between $S_{m}$ and $R_{k}$ are reciprocal. In addition, we assume that the $\mathrm{h}_{1 i, k}$ 's, $i=1,2, \ldots, M$ and $\mathrm{h}_{2 i, k}$ 's, $i=1,2, \ldots, N, k=1,2, \ldots, K$, are independent and $h_{m i, k} \sim \mathcal{C N}(0,1)$, i.e., Rayleigh fading channels. At relay $R_{k}$, we can rewrite (3) as

$$
\left[x^{\prime}, y^{\prime}\right]=\underset{x \in \mathcal{C}, y \in \mathcal{C}}{\arg \min }\left\|\mathbf{s}_{k}-\sqrt{\frac{P}{M}} \mathbf{h}_{1 k}^{T} \mathbf{w}_{1} x-\sqrt{\frac{P}{N}} \mathbf{h}_{1 k}^{T} \mathbf{w}_{1} y\right\|^{2},,
$$

Recall that the signals $x$ and $y$ are transmitted from $S_{1}$ and $S_{2}$, respectively. The estimate of $x$ and $y$ is written as $x^{\prime}$ and $y^{\prime}$, respectively. Based on pairwise error probability (PEP) analysis and assuming that all code $\mathbf{u}$ are equally likely, the average error rate is upper bounded by

$$
\begin{aligned}
\mathbf{P}_{\mathrm{MAC}}^{k} & \leq Q\left(\sqrt{\frac{\bar{\gamma}\left\|\mathbf{h}_{1 k}^{T} \mathbf{w}_{1}\left(x-x^{\prime}\right)\right\|^{2}}{2 M}}\right) \\
& +Q\left(\sqrt{\frac{\bar{\gamma}\left\|\mathbf{h}_{2 k}^{T} \mathbf{w}_{2}\left(y-y^{\prime}\right)\right\|^{2}}{2 N}}\right) \\
& \triangleq \sum_{i=1}^{2} Q\left(\sqrt{\gamma_{i b}}\right),
\end{aligned}
$$


where $\gamma_{1 b}=\frac{2 \bar{\gamma}\left\|\mathbf{h}_{1 k}^{T} \mathbf{w}_{1}\right\|^{2}}{M}$ and $\gamma_{2 b}=\frac{2 \bar{\gamma}\left\|\mathbf{h}_{2 k}^{T} \mathbf{w}_{2}\right\|^{2}}{N}$ are the equivalent system SNR of $S_{1}$ and $S_{2}$, respectively, and $Q(\cdot)$ is the Gaussian Q-function ([15], 2-1-97).

With power constraints at the sources, we minimize $\mathbf{P}_{\text {MAC }}^{k}$ subject to that the norms of $\mathbf{w}_{\mathbf{1}}$ and $\mathbf{w}_{\mathbf{2}}$ are bounded by constant, i.e., $\left\|\sqrt{\frac{P}{M}} \mathbf{w}_{1}\right\|^{2} \leq P$ and $\left\|\sqrt{\frac{P}{N}} \mathbf{w}_{2}\right\|^{2} \leq P$, respectively, which leads to $\left\|\mathbf{w}_{1}\right\|^{2} \leq M,\left\|\mathbf{w}_{2}\right\|^{2} \leq N$.

The optimal beamforming vectors can be obtained by maximizing the SNR of $S 1$ and $S_{2}$, respectively, as the objective is the sum of two monotonous Q-function expressions, and the constraints are independent of each other. Thus, the optimal beamforming vectors in the multiple-antenna two-way relay system can be easily obtained as

$$
\mathbf{w}_{1, \mathrm{opt}}=\sqrt{M} \frac{\mathbf{h}_{1 k}^{*}}{\left\|\mathbf{h}_{1 k}\right\|}, \mathbf{w}_{2, \mathrm{opt}}=\sqrt{N} \frac{\mathbf{h}_{2 k}^{*}}{\left\|\mathbf{h}_{2 k}\right\|} .
$$

Note that the results are similar to the maximum ratio transmission (MRT) [16]. It is proved that the MRT obtained from one-way relay systems is still valid in the two-way relay system $[17,18]$.

Using the optimal beamforming vectors in (12), the lower bound on $\mathbf{P}_{\text {MAC }}^{k}$ can be obtained exactly in the same way as in [19]. Assuming a genie-aided source knows the message from the other before transmission, we have

$$
Q\left(\sqrt{2 \bar{\gamma} \beta_{\mathrm{min}}^{k}}\right) \leq \mathbf{P}_{\mathrm{MAC}}^{k} \leq \sum_{m=1}^{2} Q\left(\sqrt{2 \bar{\gamma} \beta_{m}^{k}}\right)
$$

where $\beta_{\min }^{k}=\min \left(\left\|\mathbf{h}_{1 k}\right\|^{2},\left\|\mathbf{h}_{2 k}\right\|^{2}\right)$ and $\beta_{m}^{k}=\left\|\mathbf{h}_{m k}\right\|^{2}$.

\subsection{RS criterion}

The opportunistic two-way relay scheme with a low complexity in [7] achieves the better performance compared to a fully-distributed two-way relay scheme. Thus, we focus on the schemes that only one best relay is selected out of $K$ relays to decode-and-forward the signals in the second phase transmission. For tractable analysis, we use the equivalent SNR as an effective metric. When relay $R_{k}$ is selected, we define the equivalent SNR for the MAC phase at relay $R_{k}$ and the BC phase at $S_{m}, m=1,2$ as $\mathrm{SNR}_{\mathrm{MAC}^{k}} \leq \bar{\gamma} \beta_{\text {min }}^{k}$ and $\mathrm{SNR}_{\mathrm{BC}}, \mathrm{m}^{k}=\bar{\gamma} \beta_{m}^{k}$, respectively. Using the optimal beamforming, we compare two RS methods: one is an optimal beamforming and RS (O-BF$\mathrm{RS}$ ) scheme and the other is a suboptimal beamforming and RS (S-BF-RS) scheme.

1) O-BF-RS: For O-BF-RS, the destination node is to select the best relay among all the relays, which is denoted by $R_{\mathcal{R}}$. The selected relay $R_{\mathcal{R}}$ can achieve the minimum overall system SEP in (9). That is,

$$
\mathcal{R}=\underset{k=1,2, \ldots, K}{\arg \min }\left(P_{\mathrm{MAC}}^{k}+\frac{1}{2} \sum_{m=1}^{2} P_{\mathrm{BC}, m}^{k}\right) .
$$

2) S-BF-RS: The overall system performance is limited by the worst link. Thus, as a suboptimal scheme, we select the relay which minimizes the maximum SEP of all links. This can be done by selecting the relay that maximizes the minimum equivalent SNR of all links. Then, the RS criterion is given as

$\mathcal{R}=\underset{k=1,2, \ldots, K}{\arg \min \max }\left(P_{\mathrm{MAC}}^{k}, P_{\mathrm{BC}, 1}^{k}, P_{\mathrm{BC}, 2}^{k}\right)=\underset{k=1,2, \ldots, K}{\arg \max }\left(\bar{\gamma} \beta_{\min }^{k}\right)$.

Actually, the performance difference between the optimal scheme and the suboptimal scheme is negligible as the overall performance is generally decided by the worst link. This result can be verified in Section 6. However, even the performance is almost the same, the computation complexity of the two schemes are quite different. The general SEP expression which is conditioned on the instantaneous received SNR can be expressed as $\operatorname{SEP}(\gamma)=Q(\sqrt{(c \gamma)})$ [20], where $\gamma$ is the received SNR, $c$ is a constant which depends on the modulation mapping and $Q(\cdot)$ is the Gaussian Q-function ([15], 2-1-97). As a result, computing the Q-function to obtain the SEP expression and then determining the best relay for the O-BF-RS is much more complicated than that of the S-BF-RS, in which only the received SNR is needed. Thus, the S-BF-RS is more practical. Note that, for convenience, we use the BF-RS to replace the $S-B F-R S$ in the rest of this paper.

In the following section, we present the performance analysis for the BF-RS scheme in the multiple-antenna two-way relay system.

\section{Performance analysis of the BF-RS scheme}

\subsection{The bounds on the overall system SEP}

Based on the optimal beamforming design in (12) and the RS criterion in (15), we begin to analyze the SEP and the diversity gain of the BF-RS scheme. The SEPs of the MAC and $\mathrm{BC}$ phases are denoted by $P_{\mathrm{MAC}}^{\mathcal{R}}$ and $P_{\mathrm{BC}}^{\mathcal{R}}$, respectively. The SEPs are given by

$$
Q\left(\sqrt{2 \bar{\gamma} \beta_{\text {min }}^{\mathcal{R}}}\right) \leq P_{\text {MAC }}^{\mathcal{R}} \leq \sum_{m=1}^{2} Q\left(\sqrt{2 \bar{\gamma} \beta_{m}^{\mathcal{R}}}\right)
$$

and

$$
P_{\mathrm{BC}, m}^{\mathcal{R}}=Q\left(\sqrt{2 \bar{\gamma} \beta_{m}^{\mathcal{R}}}\right)
$$

Obviously, the bounds on the overall system SEP in (9), denoted by $P_{E \text { to } E}^{\mathcal{R}}$, are given as

$$
\max \left(P_{\mathrm{MAC}}^{\mathcal{R}}, \frac{1}{2} \sum_{m=1}^{2} P_{\mathrm{BC}, m}^{\mathcal{R}}\right) \leq P_{E \mathrm{to} E}^{\mathcal{R}} \leq P_{\mathrm{MAC}}^{\mathcal{R}}+\frac{1}{2} \sum_{m=1}^{2} P_{\mathrm{BC}, \mathrm{m}}^{\mathcal{R}} .
$$


It is well-known that for two positive values $a_{1}$ and $a_{2}$ of similar order, we have $a_{1} Q\left(x_{1}\right)+a_{2} Q\left(x_{2}\right) \approx$ $a_{u} Q\left(\min \left(x_{1}, x_{2}\right)\right)$ if $x_{1} \neq x_{2}$ and $a_{1} Q\left(x_{1}\right)+a_{2} Q\left(x_{2}\right)=$ $\left(a_{1}+a_{2}\right) Q(x)$ if $x_{1}=x_{2}=x$, where $u=\arg \min _{i=1,2}\left(x_{i}\right)$. Based on the derivation in [9], the upper and lower bounds on $P_{E \text { to } E}^{\mathcal{R}}$ can be obtained as

$$
Q\left(\sqrt{2 \bar{\gamma} \beta_{\text {min }}^{\mathcal{R}}}\right) \leq P_{E \mathrm{to} E}^{\mathcal{R}} \leq \frac{3}{2} Q\left(\sqrt{2 \bar{\gamma} \beta_{\mathrm{min}}^{\mathcal{R}}}\right) .
$$

\subsection{The distributions of the equivalent system SNR}

Corresponding to (19), we introduce the equivalent system SNR as $\gamma_{\min \mathcal{R}}=\bar{\gamma} \beta_{\min }^{\mathcal{R}}$ with the RS scheme. The distribution function of $\gamma_{\min \mathcal{R}}$ is required to analyze the error performance of the two-way relay system. Based on (15), we can obtain the following results.

Lemma 1. The CDF of $\gamma_{\min \mathcal{R}}$, is

$$
\begin{aligned}
F_{\gamma_{\min R}}(x)= & \sum_{q=0}^{K}\left(\begin{array}{c}
K \\
q
\end{array}\right)(-1)^{q} \sum_{m_{1}=1}^{M} \cdots \sum_{m_{q}=1}^{M} \sum_{m_{q+1}=1}^{N} \cdots \sum_{m_{2 q}=1}^{N} \\
& \times \frac{(1 / \bar{\gamma})^{\zeta_{1}}}{\beta_{1}} x^{\zeta_{1}} e^{-\left(\frac{2 q}{\bar{\gamma}}\right) x},
\end{aligned}
$$

where $\Gamma(t)=\int_{0}^{\infty x^{t-1} e^{-x} d x}$ is the Gamma function [21], $\zeta_{1}=\sum_{p=1}^{2 q}\left(m_{p}-1\right), \beta_{1}=\prod_{p=1}^{2 q}\left(m_{p}-1\right)$ !.

Proof. See Appendix 7.1.

\subsection{Average end-to-end SEP expression}

For BPSK modulation, the average SEP can be expressed as [20]

$$
\bar{P}_{E \mathrm{to} E}=E[Q(\sqrt{2 \gamma})]=\frac{1}{\sqrt{2 \pi}} \int_{0}^{\infty} e^{-\frac{t^{2}}{2}} F_{\gamma}\left(\frac{t^{2}}{2}\right) d t,
$$

where $\gamma$ is the instantaneous SNR.

Proposition 1. Applying Lemma 1 and (19) into (21), the upper and lower bounds of the average end-to-end SEP, denoted by $\bar{P}_{E \text { to } E}^{\mathcal{R}}$, are given as

$$
P_{e} \leq \bar{P}_{E \text { to } E}^{\mathcal{R}} \leq \frac{3}{2} P_{e}
$$

where

$$
\begin{aligned}
P_{e}= & \frac{1}{2 \sqrt{\pi}} \sum_{q=0}^{K}\left(\begin{array}{l}
K \\
q
\end{array}\right)(-1)^{q} \sum_{m_{1}=1}^{M} \ldots \sum_{m_{q}=1}^{M} \sum_{m_{q+1}=1}^{N} \ldots \sum_{m_{2 q}=1}^{N} \\
& \times \frac{(1 / \bar{\gamma})^{\zeta_{1}}}{\beta_{1}} \frac{\Gamma\left(\zeta_{1}+\frac{1}{2}\right)}{\left(\frac{2 q}{\bar{\gamma}}+1\right)^{\zeta_{1}+\frac{1}{2}}}
\end{aligned}
$$

Proof. See Appendix 7.1.

\subsection{Asymptotic SEP of the BF-RS scheme}

In this subsection, we derive an asymptotic SEP expression of the BF-RS scheme.

Lemma 2. The PDF of the equivalent system SNR $\gamma_{\min \mathcal{R}}$ can be approximated by polynomial terms as

$$
f_{\gamma_{\min R}}(x)=c x^{K \min (M, N)-1}+o\left(x^{K \min (M, N)-1}\right),
$$

where $c$ is a constant and if $M \neq N, c=$ $\frac{K \min (M, N)}{(\min (M, N) !)^{K} \bar{\gamma}^{K \min (M, N)}}$, otherwise $c=\frac{2^{K} K M}{(M !)^{K} \bar{\gamma}^{K M}}$.

Proof. See Appendix 7.2.

From (24) and the expression in (19), an expression for the asymptotic average SEP of the BF-RS transmitting scheme can be found in the following proposition.

Proposition 2. In high SNR regime, the asymptotic average SEP of the overall system is

$$
\bar{P}_{E \operatorname{to} E}^{R} \leq\left\{\begin{array}{c}
\frac{3 \Gamma\left(\frac{2 K \min (M, N)+1}{2}\right)}{4 \sqrt{\pi}(\min (M, N) !)^{K}} \bar{\gamma}^{-K \min (M, N)}, M \neq N \\
\frac{3 \times 2^{K-2} \Gamma\left(\frac{2 K M+1}{2}\right)}{\sqrt{\pi}(M !)^{K}} \bar{\gamma}^{-K M}, M=N
\end{array}\right.
$$

Proof. See Appendix 7.2.

According to Proposition 2, we can conclude that the diversity gain of the joint beamforming and RS scheme is $K \min (M, N)$. The BF-RS scheme achieves a full transmit and RS diversity gain.

\section{Transmit power allocation}

We have derived an asymptotic expression for the SEP of the BF-RS scheme in the last section, which was based on the assumption that the transmission power of the sources and relays are identical, i.e., $P_{1}=P_{2}=P_{r}=P$. The scheme is called as equal PA (EPA) scheme. For the case that the relays and sources are wire-powered, the total power constraint is usually adopted. In [9], an optimal power allocation scheme (OPA) have been proposed to achieve the better SEP performance. However, for the case that the relays and sources are battery-powered, we need to consider individual transmission power constraints, i.e., $0 \leq P_{1}, P_{2}, P_{r} \leq P$. Thus, in this section, we propose an individual PA (IPA) scheme under the individual transmission power constraints.

We also denote the SNRs at the relay and the sources by $\gamma_{m}=\frac{P_{m}}{\sigma^{2}}, m=1,2$, and $\gamma_{r}=\frac{P_{r}}{\sigma^{2}}$, respectively. Hence, we can rewrite (18) as 


$$
\begin{aligned}
& Q\left(\sqrt{2 \min \left(\gamma_{1} \beta_{1}^{\mathcal{R}}, \gamma_{2} \beta_{2}^{\mathcal{R}}\right)}\right) \leq P_{E \mathrm{to} E}^{\mathcal{R}} \leq \sum_{m=1}^{2} \\
& \quad \times\left(Q\left(\sqrt{2 \gamma_{m} \beta_{m}^{\mathcal{R}}}\right)+\frac{1}{2} Q\left(\sqrt{2 \gamma_{r} \beta_{m}^{\mathcal{R}}}\right)\right) .
\end{aligned}
$$

According to the derivation of (19), we can find that the SEP performance is mainly limited by the worst link in the two-way relay system. As a result, reducing the power consumption of the best link partly will hardly affect the system performance. Based on this observation, we propose an IPA scheme to save power consumption. The proposed IPA scheme is given by

$$
P_{1} \eta_{1}^{\mathcal{R}}=P_{2} \eta_{2}^{\mathcal{R}}=P_{r} \min \left(\eta_{1}^{\mathcal{R}}, \eta_{2}^{\mathcal{R}}\right),
$$

where $\eta_{m}^{\mathcal{R}}=\sqrt{\beta_{m}^{\mathcal{R}}}=\left\|\mathbf{h}_{m \mathcal{R}}\right\|, m=1,2$ or equivalently,

$$
P_{m}=\frac{P \eta_{n}^{\mathcal{R}}}{\max \left(\eta_{1}^{\mathcal{R}}, \eta_{2}^{\mathcal{R}}\right)}, \quad(m, n) \in\{1,2\} ;(m \neq n)
$$

and

$$
P_{r}=P \text {. }
$$

As in the derivation of (19), it is obvious to draw the conclusion that the IPA scheme have the same bounds as the EPA scheme, which implies that their performances would be almost the same (this is later confirmed by simulations in Section 6). Meanwhile, it can be shown that considerable transmission power saving is achieved using the IPA compared to the EPA.

We use the proposed IPA scheme in the multipleantenna two-way relay system with $M$ antennas sources and $K$ relays. The average power consumption for the IPA, normalized by the EPA, is given by

$$
\frac{\mathbb{E}\left[P_{T, \mathrm{IPA}}\right]}{\mathbb{E}\left[P_{T, \mathrm{EPA}}\right]}=\frac{2}{3}+\frac{1}{3} \mathbb{E}\left[\frac{\min \left(\eta_{1}^{\mathcal{R}}, \eta_{2}^{\mathcal{R}}\right)}{\max \left(\eta_{1}^{\mathcal{R}}, \eta_{2}^{\mathcal{R}}\right)}\right] .
$$

Since $0 \leq \frac{\min \left(\eta_{1}^{\mathcal{R}}, \eta_{2}^{\mathcal{R}}\right)}{\max \left(\eta_{1}^{\mathcal{R}}, \eta_{2}^{\mathcal{R}}\right)} \leq 1$, the total transmission power consumption of the IPA is always less than that of the EPA.

Since a closed-form solution of the total power consumption for the proposed IPA scheme is very complicated, we consider the dual case when $M=N$, since the system is more efficient on this antenna configuration. For notational convenience, we use the pair $(M, K)$ to represent the system configuration, e.g., $(2,4)$ represents that both the source nodes have $M=2$ antennas and a single relay is selected out of $K=4$ relays. We investigate the behaviors of $\mathbb{E}\left[\frac{\min \left(\eta_{1}^{\mathcal{R}}, \eta_{2}^{\mathcal{R}}\right)}{\max \left(\eta_{1}^{\mathcal{R}}, \eta_{2}^{\mathcal{R}}\right)}\right]$ when $(M, K)=(1,1)$, $(M, K)=\left(M \in \mathbb{N}^{+}, \infty\right)$, and $(M, K)=(\infty, K \in \mathbb{N})$ in Lemma 3 and Proposition 3 to gain insights into the performance analysis.
Lemma 3. We consider the BF-RS scheme with the IPA in the two-way relay system. When $(M, K)=(1,1)$, for two RVs $X=\eta_{1}^{\mathcal{R}}$, and $Y=\eta_{2}^{\mathcal{R}}$, we have the CDF of $Z=$ $\frac{\min (X, Y)}{\max (X, Y)}$ as

$$
F_{Z}(z)=2\left(1-\frac{1}{1+z^{2}}\right) .
$$

Proof. See Appendix 7.3.

From Lemma 3, the expectation of $Z$ is obtained by $\mathbb{E}[Z]=\int_{0}^{1} z f_{Z}(z) d z=F_{Z}(1)-\int_{0}^{1} F_{Z}(z) d z=\frac{\pi}{2}-1$. The normalized power consumption for the IPA in (29) becomes 0.8569 . The result indicates that the IPA could save about $14 \%$ transmission power consumption compared to the EPA.

Proposition 3. We consider the BF-RS scheme with the IPA in the two-way relay system. When $(M, K)=$ $\left(M \in \mathbb{N}^{+}, \infty\right)$ and $(M, K)=\left(\infty, K \in \mathbb{N}^{+}\right)$, for two RVs $X=\eta_{1}^{\mathcal{R}}$ and $Y=\eta_{2}^{\mathcal{R}}$, it can be shown that the expectation of $Z=\frac{\min (X, Y)}{\max (X, Y)}$ becomes 1, i.e., $\mathbb{E}(Z)=1$.

\section{Proof. See Appendix 7.4.}

As shown in Section 6, a better power saving can be achieved by decreasing the numbers of the sources antennas and the relays. It indicates the IPA is more suitable to be applied in the scenarios where the numbers of sources antennas and relays are limited.

At the end of this section, we consider the complexity order of the proposed IPA schemes in the multi-antenna two-way relay system. Note that the complexity order is dominated by complex multiplications in this paper. For Eq. (27), the calculations include the 2 norm operations, additions, comparisons, and divisions. As a result, the complexity order of the IPA with $M$-antennas and $N$ antennas at $S_{1}$ and $S_{2}$, respectively, is $O\left(M^{2}\right)+O\left(N^{2}\right)+$ $O(M)+O(N)+O(1)=O\left(\max (M, N)^{2}\right)$.

\section{Simulation results}

In this section, we evaluate the performances of the BFRS and the IPA schemes. BPSK modulation is used and the i.i.d. Rayleigh flat fading channels are assumed in all the simulations. We assume that the transmission power of the sources and the relays are identical, i.e., $P_{1}=P_{2}=$ $P_{r}=1(0 \mathrm{~dB})$, in the simulations of Figs. 2, 3, 4, 5 and 6. For convenience of the comparison, we assume that the total power constraint of each PA scheme is $P_{T}=3(=4.77$ $\mathrm{dB})$. Furthermore, we assume that the sources and relays have the same noise variance.

Besides, we introduce the curves obtained by using $(\mathrm{SNR}-a)^{-d}$ as the references to analyze the diversity gain, where $a$ is a real number and $d$ is a positive integer. 


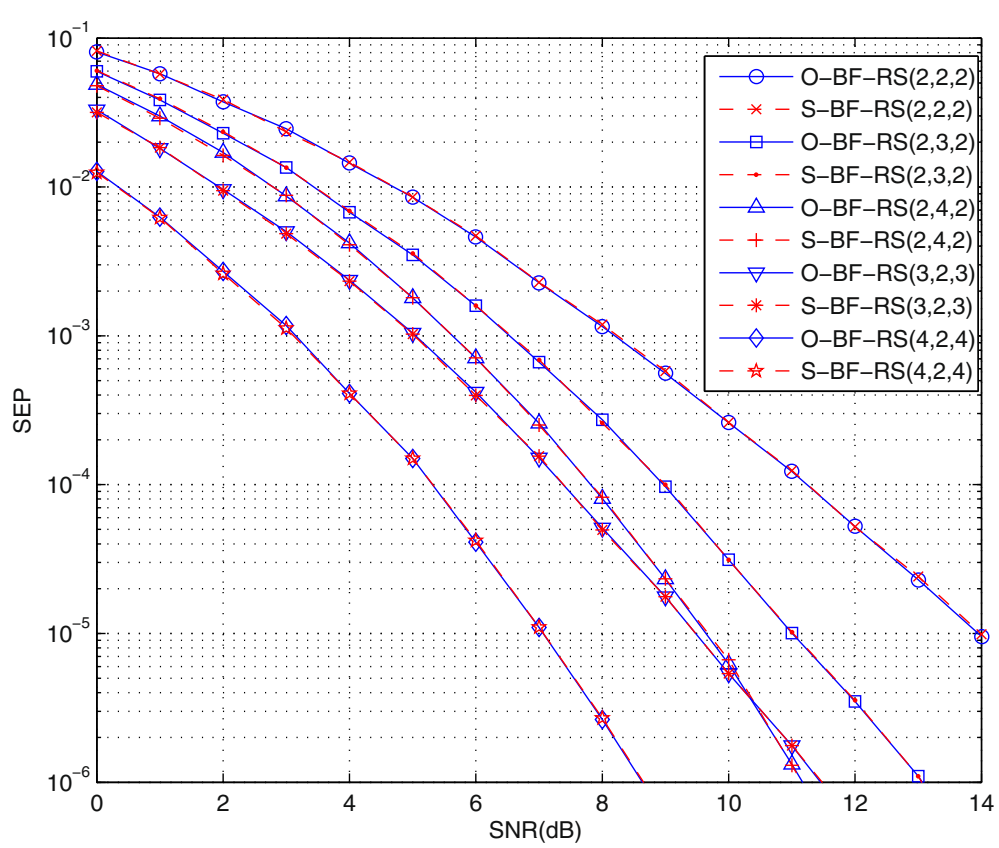

Fig. 2 BER performance comparison of O-BF-RS and S-BF-RS, where $P_{1}=P_{2}=P_{r}=1$

If the SEP curves of the schemes tend to be parallel with $(\mathrm{SNR}-a)^{-d}$ in high SNR region, it reveals that a diversity gain of $d$ is achieved.

\subsection{Simulated results}

In Fig. 2, we compare the performance of the O-BF-RS and S-BF-RS schemes, where $P_{1}=P_{2}=P_{r}=1$. It is shown that the performance difference between the S-BF$\mathrm{RS}$ and the O-BF-RS schemes is negligible. The intuitive explanation of the phenomenon is that the overall system performance of the two way relay system is limited by the worst link. In other words, once the relay which maximizes the worst SNR of the two links is selected, it is equivalent to minimize the overall system SEP. Thus,

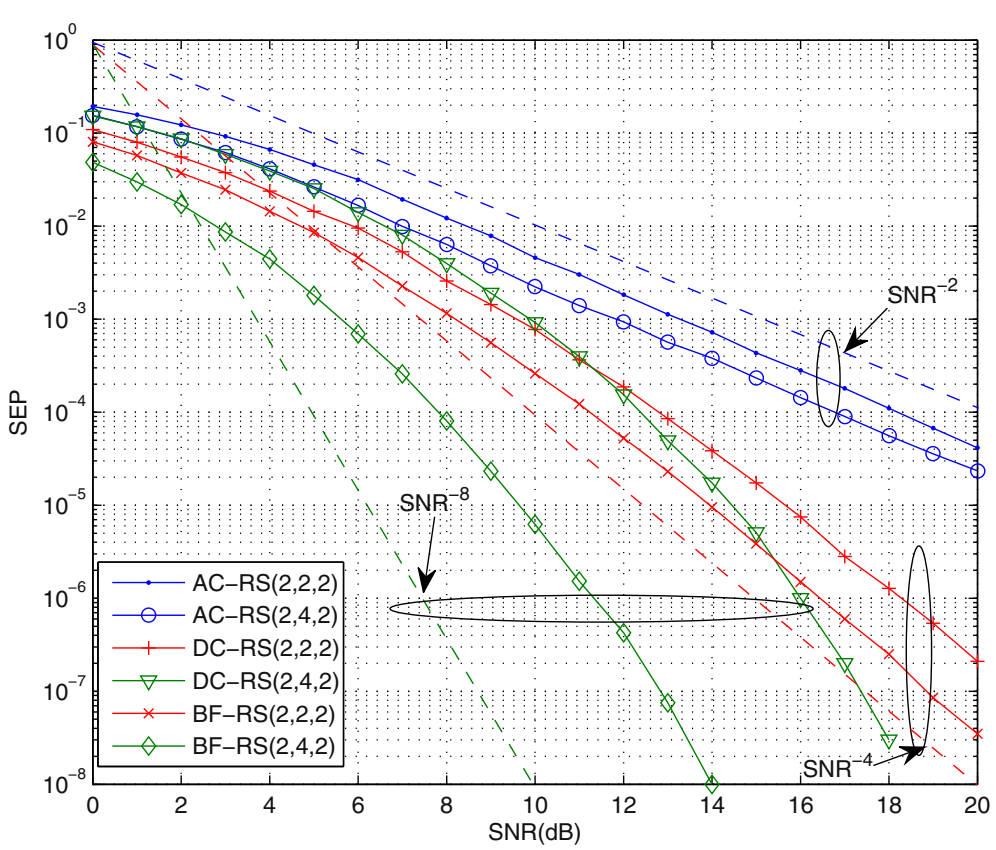

Fig. 3 BER performance comparison of different transmission schemes, where $P_{1}=P_{2}=P_{r}=1$ 


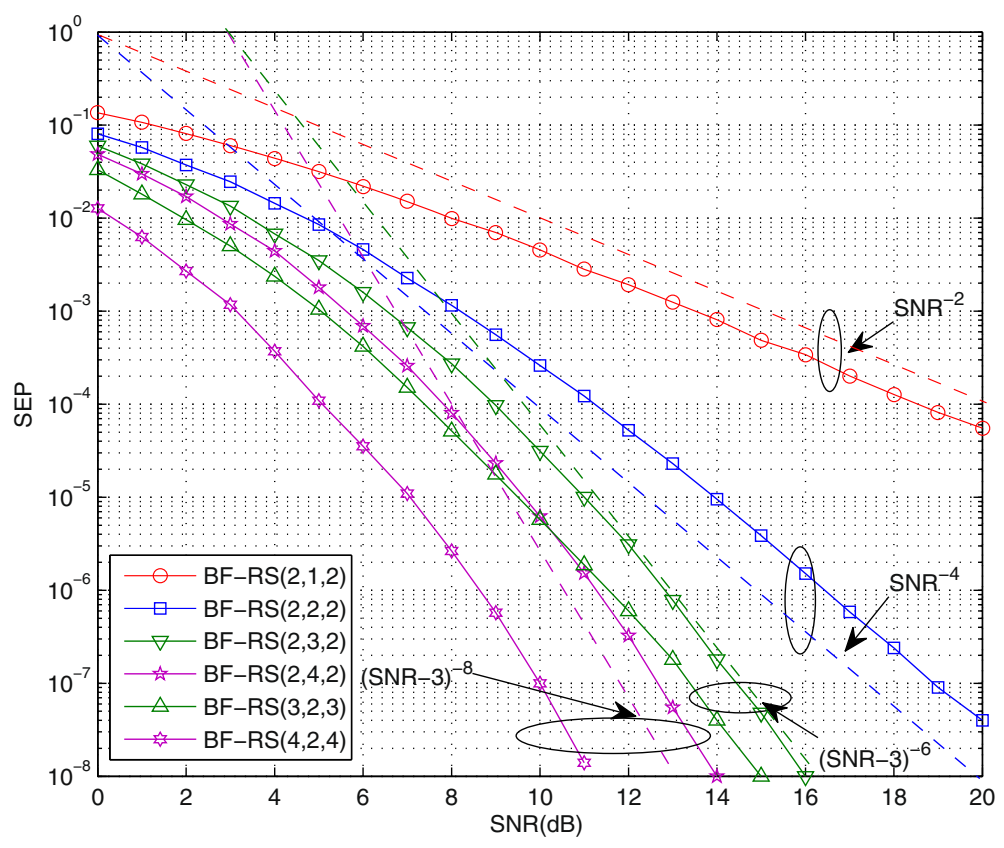

Fig. 4 The achieved diversity gain of the BF-RS scheme with different sources antennas and relays configurations, where $P_{1}=P_{2}=P_{r}=1$

the result indicates that we can use the S-BF-RS instead of the O-BF-RS. [...] The figure also presents that the curves of the setting $(2,4)$ and $(3,2)$ intersect each other. The qualitative explanation of the phenomenon is that once the SNR is low, more antennas is beneficial to achieving the better performance. Thus, the performance of $(3,2)$ outperforms that of $(2,4)$ in low SNR regions. However, since the diversity of $(2,4)$ is larger than the diversity of $(3,2)$, the performance of $(2,4)$ outperforms that of $(3,2)$ in high SNR regions, which further results in the intersection of the curves.

Figure 3 shows the overall system SEP performance of different transmission schemes to validate the advantages of the BF-RS scheme. We compare the BF-RS scheme with

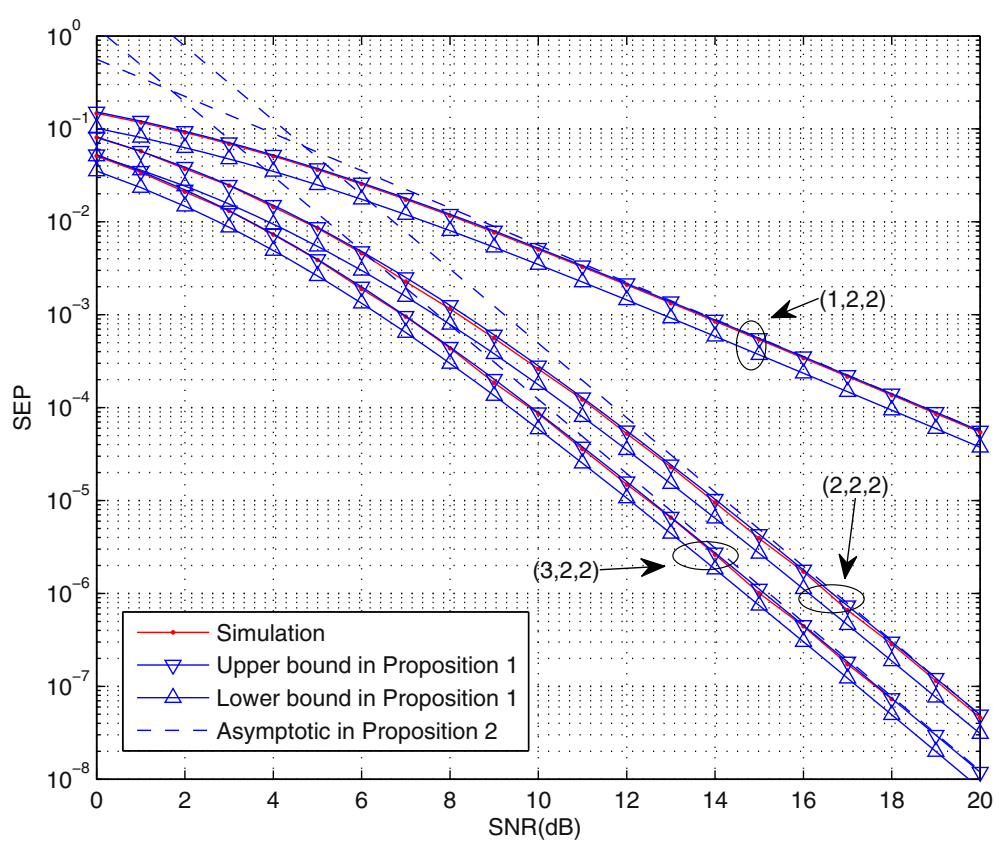

Fig. 5 SEP comparison of analytical and simulated results, where $P_{1}=P_{2}=P_{r}=1$ 


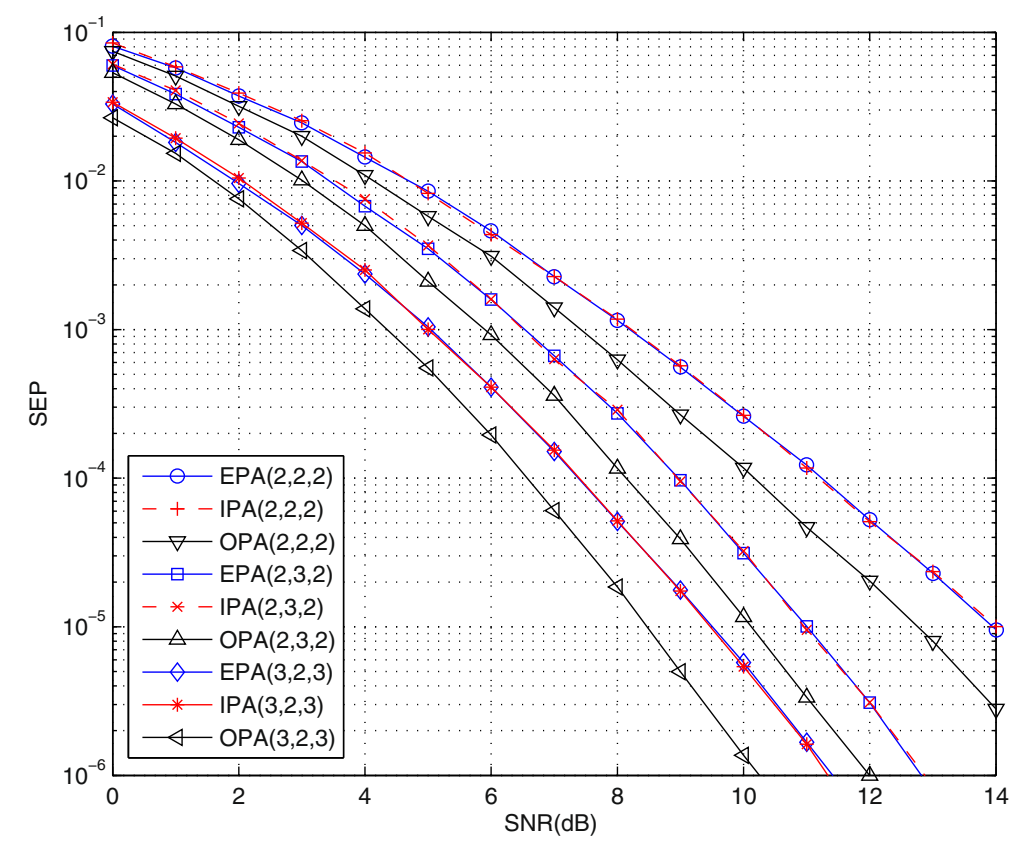

Fig. 6 SEP performance comparison of the proposed IPA scheme, the EPA scheme and the OPA scheme

the Almouti code RS (AC-RS) and the diagonal code RS (DC-RS) with $M=2$. Note that the AC-RS scheme also requires the full CSI for the relay selection process. It is shown that both the DC-RS and BF-RS schemes can achieve a full transmit and RS diversity gain of $d=K M$. However, the diversity order of the AC-RS scheme is only $d=M$. Comparing the SEP curves of AC-RS between $(2,2)$ and $(2,4)$, we can find that the RS is only helpful for coding gain, but not diversity gain in the AC-RS scheme. Overall, the BF-RS scheme outperforms the AC-RS and DC-RS schemes in all the SNR regions.

\subsection{Analytical results}

In Fig. 4, we illustrate the diversity gain of the BF-RS scheme with different configurations of $(M, K)$. The performance curves of the BF-RS scheme show the same decay trend with that of $\mathrm{SNR}^{-\mathrm{KM}}$ for all the configurations. The results show that the BF-RS scheme achieves a diversity gain of $d=K M$. In addition, we can find that the performance of configurations of $(M, K)=(3,2)$ and $(4,2)$ are better than those of configurations of $(2,3)$ and $(2,4)$, respectively. From this, we can draw the conclusion that increasing the number of the source antennas is more helpful than increasing the number of the relays on the overall system SEP performance.

Figure 5 shows the SEP performance of a special case of the BF-RS scheme with $(M, K, N)=(1,2,2),(2,2,2)$, and $(3,2,2)$. The simulation result is within the curves of the theoretical upper and lower bounds. As shown in Fig. 5, the upper bound is very close to the simulated
SEP curve. The accuracy of upper bound can indicate that the approximations in the theoretical derivation of (19) are accurate. Meanwhile, it can be noticed that the lower bound is parallel with the simulated SEP curve with a constant gap in all SNR region. In summary, our derived bounds can predict the performance accurately. Furthermore, the analytical asymptotic SEP and simulated SEP of the BF-RS scheme are presented as well. It is shown that, at the high SNR region, the asymptotic SEP given by (25) approaches the simulated SEP. The result has verified our analytical derivation.

\subsection{PA}

Figure 6 shows the SEP performance of the BF-RS scheme with the proposed IPA scheme. The results of the EPA and OPA [9] are also provided for comparison purposes. It can be observed that the SEP curves of the proposed IPA scheme are very close to the SEP curves of the EPA scheme. Meanwhile, it is shown that the gap between the performances of OPA and IPA is about $1-2 \mathrm{~dB}$. This gain results from the fact that the more power consumption is used in OPA than that in IPA.

In Fig. 7, we compare the power consumption by the IPA and EPA schemes. The power cost of the EPA is the same as that of the OPA, i.e., $P_{T}=3$. It can be observed that the proposed IPA scheme obtains almost $14 \%$ power saving when $(M, K)=(1,1)$. The power cost increases with the number of source antennas and the number of relays. The results verify our derivation. In summary, the EPA is introduced as a benchmark in Figs. 6 and 7. The results 


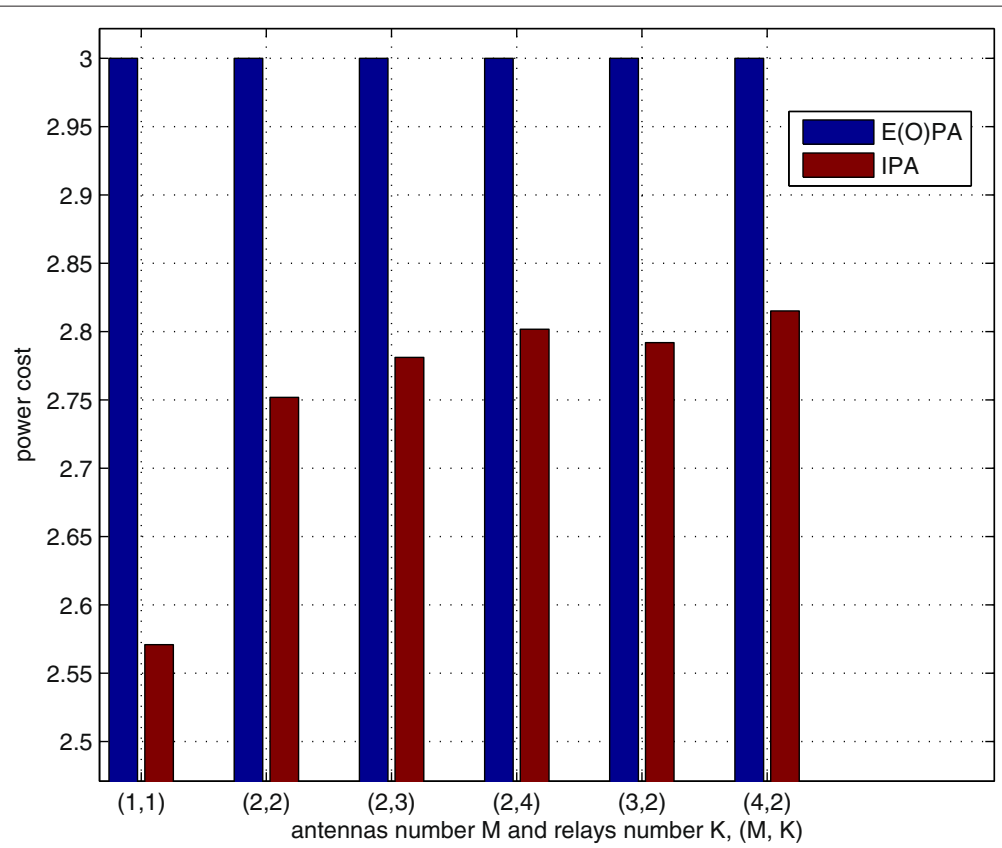

Fig. 7 Simulated transmission power consumption of the proposed IPA scheme, the EPA scheme, and the OPA scheme

show that the OPA outperforms the EPA in terms of the SEP once the total power constraint is adopted. Likewise, the IPA outperforms the EPA in terms of the power cost once the independent power constraint is adopted.

In Fig. 8, we plot the curved surface of the expectation of $Z$ in terms of different numbers of source antennas and relays. It is shown that the $\mathbb{E}[Z]$ increases monotonically, which confirms that the effective power saving of the proposed IPA scheme is obtained when the numbers of antennas of the sources and the relays are limited.

\section{Conclusions}

In this paper, we have studied the performance of various transmission schemes for multiple-antenna two-way relay systems with RS in terms of SEP. Specifically, we have focused on the performance of the BF-RS scheme as it

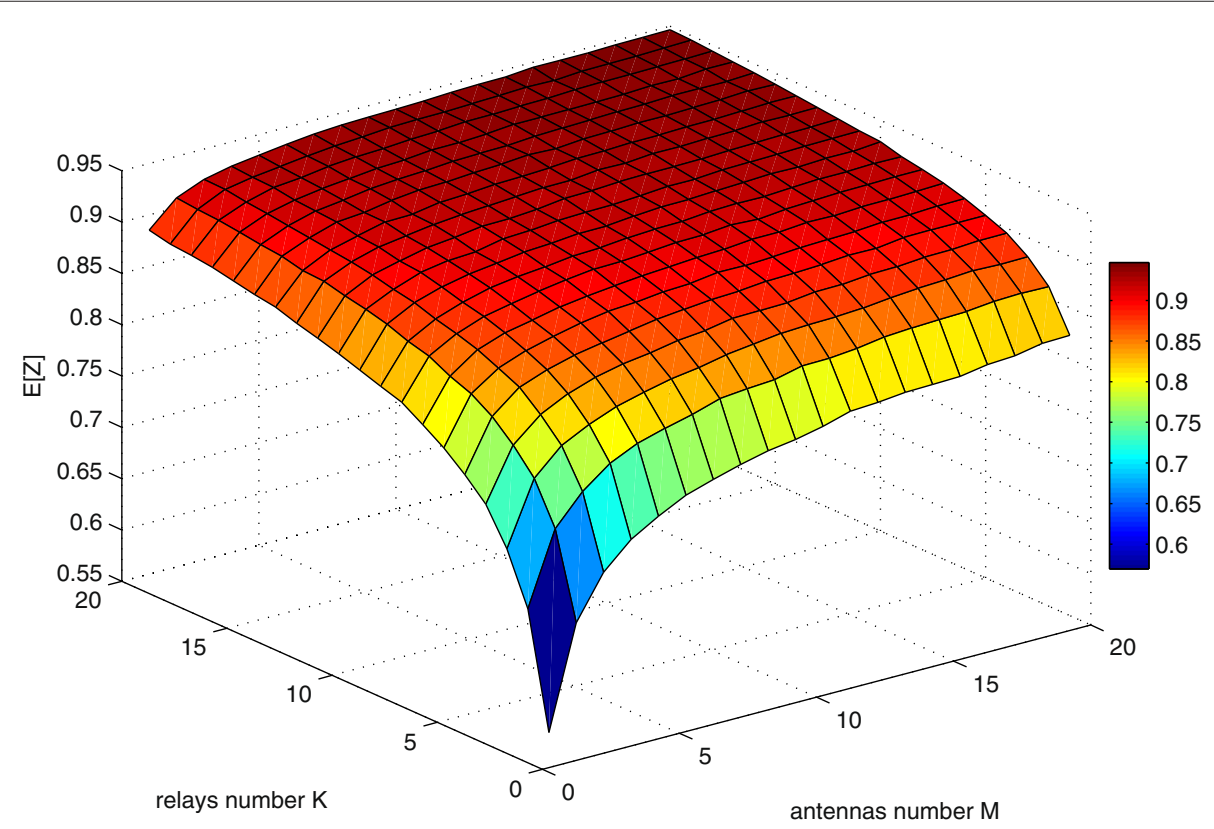

Fig. 8 Simulated expectation of $Z$ with antenna number $M$ and relay number $K$ 
can outperform other schemes significantly. We derived the analytical upper and lower bounds of the end-to-end system SEP in the closed form as well as the asymptotic SEP expression in high SNR regime. The analytical performance predictions with the derived SEP expressions were verified by simulations. Based on individual power constraints, we also proposed an IPA scheme to save the total transmit power. Both analytical and simulated results indicated that the BF-RS scheme with IPA could save considerable amount of energy.

\section{Endnote}

${ }^{1}$ We do not provide existing schemes' performances in detail in this paper, but their performances are shown in Section 6 by simulations.

\section{Appendix}

\subsection{Proof of Lemma 1, Proposition 1}

Appendix 7.1. We focus on the PDF of $\gamma_{\min \mathcal{R}}$. Let $\gamma_{m k}=$ $\gamma\left\|\mathbf{h}_{m k}\right\|^{2}, m=1,2$ and $\mathbf{h}_{1 k}=\left[\begin{array}{llll}h_{11, k} & h_{12, k} & \ldots & h_{1 M, k}\end{array}\right]^{T}$, and $\mathbf{h}_{2 k}=\left[h_{21, k} h_{22, k} \ldots h_{2 N, k}\right]^{T}$. Since $\left|h_{1 n_{t}, k}\right|^{2}, n_{t}=$ $1,2, \ldots, M, k=1,2, \ldots, K$, is independent chi-square distributed with $2 M$ degrees of freedom, the PDF of $\gamma_{1 k}$ is $f_{\gamma_{1 k}}(\xi)=\frac{\xi^{M-1}}{(M-1) ! \gamma^{M}} e^{-\frac{\xi}{\gamma}}$. The CDF of $\gamma_{1 k}$ is given by $F_{\gamma_{1 k}}(\xi)=1-e^{-\frac{\xi}{\gamma}} \sum_{q=0}^{M-1} \frac{(\xi / \gamma)^{q}}{q !}$. Likewise, the PDF and CDF of $\gamma_{2 k}$ can be easily obtained as well. Define $\beta_{\text {min }}^{k}=\min \left(\gamma_{1 k}, \gamma_{2 k}\right)$, the CDF of $\beta_{\text {min }}^{k}$ is $F_{\beta_{\text {min }}^{k}}(\xi)=$ $1-\left(e^{-\frac{2 \xi}{\gamma}} \sum_{m_{1}=0}^{M-1} \sum_{m_{2}=0}^{N-1} \frac{(\xi / \gamma)^{m_{1}+m_{2}}}{m_{1} ! m_{2} !}\right)$.

Using order statistics, the CDF of the equivalent system $\operatorname{SNR} \gamma_{\min \mathcal{R}}=\max _{k=1,2, \ldots, K} \bar{\gamma} \beta_{\min }^{k}$ is

$F_{\gamma_{\min R}}(x)=\left(F_{\beta_{\min }^{k}}(x)\right)^{K}=\left[1-\left(e^{-\frac{2 \xi}{\gamma}} \sum_{m_{1}=0}^{M-1} \sum_{m_{2}=0}^{N-1} \frac{(\xi / \gamma)^{m_{1}+m_{2}}}{m_{1} ! m_{2} !}\right)\right]^{K}$.

Consequently, using the binomial expansion, we can obtain the CDF of $\gamma_{\min \mathcal{R}}$. The PDF of $\gamma_{\min } \mathcal{R}$ can be obtained by differentiating the CDF in (31).

Applying Lemma 1 in (19) and (21), the analytical endto-end SEP in Proposition 2 can be obtained by the integral of $[22,2.33 .10,8.350 .2]$.

\subsection{Proof of Lemma 2, Proposition 2}

Appendix 7.2. Differentiating the CDF in (31), we have

$$
f_{\gamma_{\min \mathcal{R}}}(x)=K\left(F_{\beta_{\min }^{k}}(x)\right)^{K-1} f_{\beta_{\min }^{k}}(x) .
$$

According to the Taylor series expansion formula and the limit theory, we can approximate the PDF by polynomial terms as

$$
f_{\gamma_{\min R}}(x)=c x^{K \min (M, N)-1}+o\left(x^{K \min (M, N)-1}\right),
$$

where $c$ is a constant and if $M \neq N, c=$ $\frac{K \min (M, N)}{(\min (M, N) !)^{K} \bar{\gamma}^{K \min (M, N)}}$, otherwise $c=\frac{2^{K} K M}{(M !)^{K} \bar{\gamma}^{K M}}$.

By (19), the SEP of the overall system is upper bounded by

$$
P_{E \text { to } E}^{\mathcal{R}} \leq \frac{3}{2} Q\left(\sqrt{2 \gamma \beta_{\text {min }}^{\mathcal{R}}}\right)=\frac{3}{2} Q\left(\sqrt{2 \gamma_{\min \mathcal{R}}}\right) .
$$

Applying Lemma 2 in [23], the average SEP of the overall system is given by

$$
\begin{aligned}
\bar{P}_{E \mathrm{to} E}^{\mathcal{R}} & \leq \frac{3}{2} \int_{0}^{\infty} Q(\sqrt{2 x}) f_{\gamma_{\min R}}(x) d w \\
& =\frac{3}{2} \int_{0}^{\infty} \int_{\sqrt{2 x}}^{\infty} \frac{1}{\sqrt{2 \pi}} e^{-\frac{z^{2}}{2}}\left[c x^{K \min (M, N)-1}+o\left(x^{K \min (M, N)-1}\right)\right] d z d x \\
& =\frac{3 c}{2 \sqrt{2 \pi}} \int_{0}^{\infty} e^{-\frac{z^{2}}{2}} \int_{0}^{\frac{z^{2}}{2}}\left[c x^{K \min (M, N)-1}+o\left(x^{K \min (M, N)-1}\right)\right] d x d z \\
& =\left\{\begin{array}{c}
\frac{3 \Gamma\left(\frac{2 K \min (M, N)+1}{2}\right)}{4 \sqrt{\pi}\left(\min (M, N) ! !^{K}\right.} \bar{\gamma}^{-K \min (M, N)}, M \neq N \\
\frac{3 \times 2^{K-2} \Gamma\left(\frac{2 K M+1}{2}\right)}{\sqrt{\pi}(M !)^{K}} \bar{\gamma}^{-K M}, M=N
\end{array}\right.
\end{aligned}
$$

where $\Gamma(\cdot)$ is the Gamma function.

\subsection{Proof of Lemma 3}

Since $X=\eta_{1}^{\mathcal{R}}$ and $Y=\eta_{2}^{\mathcal{R}}$ are RVs with generalized Rayleigh distribution $[15,2-1-136]$. When $(M, K)=(1,1)$, the CDFs of $X$ and $Y$ are given by $F_{X}(x)=1-e^{-\frac{x^{2}}{2}}$ and $F_{Y}(y)=1-e^{-\frac{y^{2}}{2}}$, respectively. The CDF of $Z=\frac{\min (X, Y)}{\max (X, Y)}$ can be expressed as

$$
F_{Z}(z)=\underbrace{\operatorname{Pr}\{Z \leq z, X \leq Y\}}_{\triangleq I_{1}}+\underbrace{\operatorname{Pr}\{Z \leq z, X \geq Y\}}_{\triangleq I_{2}} .
$$

For $x, y \geq 0$, we have

$$
\begin{aligned}
I_{1} & =\operatorname{Pr}\left\{\frac{X}{Y} \leq z, X \leq Y\right\} \\
& =\int_{0}^{\infty} \operatorname{Pr}\{X \leq y z, X \leq y\} f_{Y}(y) d y \\
& =\int_{0}^{\infty}\left(1-e^{\frac{-(y z)^{2}}{2}}\right) y e^{\frac{-(y)^{2}}{2}} d y \\
& =1-\frac{1}{2} \int_{0}^{\infty} e^{-\left(\frac{z^{2}+1}{2}\right) t} d t \\
& =1-\frac{1}{z^{2}+1} .
\end{aligned}
$$

Due to the symmetry, it is straightforward to obtain $I_{2}$ from $I_{1}$. Then, the CDF $F_{Z}(z)=I_{1}+I_{2}=2 I_{1}$.

\subsection{Proof of Proposition 3}

Based on the RS criterion as (15), when $K \rightarrow \infty$, it is obvious to see $\lim _{K \rightarrow \infty} \mathbb{E}[X]=\infty$ and $\lim _{K \rightarrow \infty} \mathbb{E}[Y]=\infty$. Let 
$\zeta=\min (X, Y), \psi=\max (X, Y)$. Without loss of generality, we assume $X \leq Y$. As the PDF of $\psi$ is given by $f_{\psi}(y)=2 F(y) f(y)$, the condition probability density function of $\zeta$ becomes $f_{\zeta}(x \mid y)=\frac{f(x, y)}{f_{\psi}(y)}=\frac{f(x)}{2 F(y)}$.

The expectation of $Z=\frac{\min (X, Y)}{\max (X, Y)}$, when $K \rightarrow \infty$, can be expressed as

$$
\lim _{K \rightarrow \infty} \mathbb{E}[Z]=\underbrace{\lim _{X, Y \rightarrow \infty} \mathbb{E}\left[\frac{X}{Y}, X \leq Y\right]}_{\triangleq I_{3}}+\underbrace{\lim _{X, Y \rightarrow \infty} \mathbb{E}\left[\frac{Y}{X}, Y \leq X\right]}_{\triangleq I_{4}} .
$$

Thus, we have

$$
\begin{aligned}
I_{3} & =\lim _{x, y \rightarrow \infty} \int_{0}^{y} \frac{x}{y} \cdot f_{\zeta}(x \mid y) d x \\
& =\lim _{x, y \rightarrow \infty} \frac{\int_{0}^{y} x f(x) d x}{2 y F(y)} \\
& =\lim _{x, y \rightarrow \infty} \frac{1}{2} \cdot \frac{y f(y)}{y f(y)+F(y)} .
\end{aligned}
$$

Clearly, with $\lim _{y \rightarrow \infty} F(y)=1$, we have $I_{3}=\frac{1}{2}$. Due to symmetry, it is straightforward to obtain $I_{4}$ for $I_{3}$ by exchange $x$ and $y$. The expectation of $Z$, when $K \rightarrow \infty$, is $\lim _{K \rightarrow \infty} \mathbb{E}[Z]=1$.

Let us now consider the expectation when $M \rightarrow \infty$ and $K \in N^{+}$. Since $X=\eta_{1}^{\mathcal{R}}$ and $Y=\eta_{2}^{\mathcal{R}}$ are generalized Rayleigh RVs, $X^{2}$ and $Y^{2}$ are chi-square distributed with $2 M$ degrees of freedom. Using high-order moments of the generalized Rayleigh distribution [15, 2-1-138] and Stirling's formula [24], we find

$$
\begin{aligned}
\mathbb{E}[X] & =\frac{\sqrt{2} \Gamma\left(M+\frac{1}{2}\right)}{\Gamma(M)} \\
& =\frac{\sqrt{2}(2 M) ! \sqrt{\pi}}{M !(M-1) ! 4^{M}} \\
& =\frac{\sqrt{4 \pi^{2} 2 M}\left(\frac{2 M}{e}\right)^{2 M}}{\sqrt{4 \pi^{2} M(M-1)}\left(\frac{M}{e}\right)^{M}\left(\frac{M-1}{e}\right)^{M-1}} \\
& =\sqrt{2(M-1)}
\end{aligned}
$$

and $\mathbb{E}\left[X^{2}\right]=\frac{2 \Gamma(M+1)}{\Gamma(M)}=2 M$. Therefore, the coefficient of variation for $X$ is obtained as $\lim _{M \rightarrow \infty} \frac{\mathbb{E}\left[X^{2}\right]-\mathbb{E}[X]^{2}}{\mathbb{E}[X]}=0$. The result indicates that $X$ is equal to $\sqrt{2(M-1)}$ with probability 1 when $M \rightarrow \infty$. The derivation is also valid to $Y$. The expectation of $Z=\frac{\min (X, Y)}{\max (X, Y)}$, when $M \rightarrow \infty$, can be expressed as

$$
\lim _{M \rightarrow \infty} \mathbb{E}[Z]=\lim _{M \rightarrow \infty} \frac{\sqrt{2(M-1)}}{\sqrt{2(M-1)}}=1 .
$$

It completes the proof.

\section{Competing interests}

The authors declare that they have no competing interests.

\section{Acknowledgements}

This work is supported in part by the National Science Foundation of China (NSFC, Grant No. 61471008), the Specialized Research Fund for the Doctoral Program of the Ministry of Education of China (Grant No. 20120001120125), and the National Natural Science Foundation of Beijing (Grant No. 4144075). The correspondence author is Chen Chen (E-mail: c.chen@pku.edu.cn).

\section{Author details}

${ }^{1}$ State Key Laboratory of Advanced Optical Communication Systems and Networks, Peking University, 100871 Beijing, China. ${ }^{2}$ Representative Office in Chengdu of Information Department, 610041 Chengdu, China.

Received: 16 January 2015 Accepted: 6 July 2015

Published online: 23 July 2015

\section{References}

1. T Cui, F Gao, T Ho, A Nallanathan, in Proc. IEEE ICC. Distributed space-time coding for two-way wireless relay networks (IEEE Beijing, China, 2008), pp. 3888-3892

2. D To, J Choi, IM Kim, Error probability analysis of bidirectional relay systems using Alamouti scheme. IEEE Commun. Lett. 14(8), 758-760 (2010)

3. FK Gong, JK Zhang, G Jian-hua, Distributed concatenated Alamouti codes for two-way relaying networks. IEEE Wireless Commun. Lett. 1(3), 197-200 (2012)

4. C Chen, L Bai, B Wu, J Choi, Relay selection and beamforming for cooperative bi-directional transmissions with physical layer network coding. IET Commun. 5(14), 2059-2067 (2011)

5. X Jia, L Yang, H Fu, Tight performance bounds for two-way opportunistic amplify-and-forward wireless relaying networks with TDBC protocols. EURASIP J. Wirel. Commun. Netw. 1(192), 1-8 (2012)

6. LS Jayasinghe, N Rajatheva, M Latva-Aho, Robust precoder-decoder design for physical layer network coding-based MIMO two-way relaying system. EURASIP J. Wirel. Commun. Netw. 1(137), 1-16 (2013)

7. Q Zhou, Y Li, F Lau, B Vucetic, Decode-and-forward two-way relaying with network coding and opportunistic relay selection. IEEE Trans. Commun. 58(11), 3070-3076 (2010)

8. S Beygi, M Kafashan, H Bahrami, T Le-Ngoc, M Maleki, Space-time trellis codes for two-way relay MIMO channels with single-antenna relay nodes. IEEE Trans. Veh. Technol. 62(8), 4040-4045 (2013)

9. T Do, J Wang, L Song, Y Kim, Joint relay selection and power allocation for two-way relaying with physical layer network coding. IEEE Commun. Lett. 17(2), 301-304 (2013)

10. L Song, Relay selection for two-way relaying with amplify-and-forward protocols. IEEE Trans. Veh. Technol. 60(4), 1954-1959 (2011)

11. C Zhang, J Ge, J Li, Y Hu, Fairness-aware power allocation for asymmetric two-way AF relaying networks. Electron. Lett. 48(15), 959-961 (2012)

12. S Talwar, Y Jing, S Shahbazpanahi, Joint relay selection and power allocation for two-way relay networks. IEEE Signal Process. Lett. 18(2), 91-94 (2011)

13. S Alamouti, A simple transmit diversity technique for wireless communications. IEEE J. Sel. Areas Commun. 16(8), 1451-1458 (1998)

14. EG Larsson, P Stoica, Space-time block coding for wireless communications. (Cambridge University Press, New York, 2003)

15. J Proakis, Digital communications, 4th edition. (McGraw-Hill, New York, 2000)

16. TKY Lo, Maximum ratio transmission. IEEE Trans. Commun. 47(10), 1458-1461 (1999)

17. RHY Louie, Y Li, HA Suraweera, B Vucetic, Performance analysis of beamforming in two hop amplify and forward relay networks with antenna correlation. IEEE Trans. Wireless Commun. 8(6), 3132-3141 (2009)

18. Y Lu, N Yang, H Dai, X Wang, Opportunistic decode-and-forward relaying with beamforming in two-wave with diffuse power fading. IEEE Trans. Veh. Technol. 61(7), 3050-3060 (2012)

19. M Ju, IM Kim, Error performance analysis of BPSK modulation in physical-layer network-coded bidirectional relay networks. IEEE Trans. Commun. 58(10), 2770-2775 (2010) 
20. M Soysa, HA Suraweera, C Tellambura, HK Garg, Partial and opportunistic relay selection with outdated channel estimates. IEEE Trans. Commun. 60(3), 840-850 (2012)

21. M Abramowitz, IA Stegun, Handbook of mathematical functions with formulas, graphs, and mathematical tables, 9th Edition. (Dover, New York, 1970)

22. IS Gradshteyn, IM Ryzhik, Table of integrals, series, and products, 7th edition (Academic, New York, 2007)

23. Z Wang, G Giannakis, A simple and general parameterization quantifying performance in fading channels. IEEE Trans. Commun. 51(8), 1389-1398 (2003)

24. RB Paris, D Kaminsky, Asymptotics and the Mellin-Barnes integrals. (Cambridge University Press, New York, 2001)

\section{Submit your manuscript to a SpringerOpen ${ }^{\mathcal{O}}$ journal and benefit from:}

- Convenient online submission

- Rigorous peer review

- Immediate publication on acceptance

- Open access: articles freely available online

- High visibility within the field

- Retaining the copyright to your article

Submit your next manuscript at $\gg$ springeropen.com 